\title{
A method for improving consistency in photometric databases
}

\author{
Felipe Hernández-Rodríguez \\ felipe.hernandez@cinvestav.edu.mx \\ Mario Castelán \\ mario.castelan@cinvestav.edu.mx
}

Robotics and Advanced Manufacturing Centro de Investigación y de Estudios Avanzados del I.P.N.

Ramos Arizpe, Coah., México.

\begin{abstract}
Building photometric databases usually requires the gathering of images of a still object under different light source directions. During this process, unexpected artifacts such as noise, shadows, inter-reflections and other unwanted effects introduced by the sensibility of the camera may appear along the database, diminishing its consistency as a whole and therefore its suitability for the purposes of photometric analysis. This paper describes a method for improving photometric consistency in image databases acquired under photometric rigs. The main idea of our approach is to build and analyze a luminance matrix storing the reflectance behavior of each pixel under the different light source directions. To this end, we propose to fit sinusoidal functions to the singular vectors of this luminance matrix in order to improve its agreement with Lambertian reflectance. Experiments demonstrate that our method improves the photometric consistency of the database, providing stability for the purposes of photometric analysis of the database and surface shape recovery.
\end{abstract}

\section{Introduction}

Estimating 3D shape and reflectance from imagery is a relevant topic in computer vision and computer graphics, since it simplifies the task of modeling the appearance of objects. Techniques based on photometric changes, such as the photometric stereo method (PSM), have proved to be useful for such task by enforcing illumination variations over a still object. A quick review in the area reveals that issues such as the strategic placement of light sources $[\square$, 四], the modeling of Bidirectional Reflectance Distribution Functions (BRDF) to handle non-Lambertian reflectance [ $[$ ] or shaded regions [ $\square, \square]$, and the statistical properties of photometric databases [ $[$ ] have received major attention in the field.

Similarly, approaches such as the photometric sampling [ $\square$ ] have also been developed in order to recover shape and reflectance properties through photometric features. The photometric sampling consists of measuring the response of a still object while a light source, placed on a turntable, moves around a single circular path. Research efforts in photometric sampling have been mostly focused on the recovery of surface normals by sine fitting, where the key idea is to adjust a sinusoidal function to the pixel's response along the light source trajectory. Sine fitting has shown a benefit in determining the azimuth angles of the surface normals as well as in determining specular areas $[\square, \square]$. 
Regardless of the approach, however, during the database acquisition process, unexpected artifacts such as noise, shadows, inter-reflections and other unwanted effects introduced by the sensibility of the camera may appear along the captured images. As a consequence, the suitability and consistency of the database for the purposes of photometric analysis is diminished.

This paper describes a method for improving photometric consistency in image databases acquired under photometric rigs. The main idea of our approach is to build and analyze a luminance matrix storing the reflectance behavior of each pixel under the different light source directions. To this end, we fit sinusoidal functions to the singular vectors of the luminance matrix in order to improve its agreement with Lambertian reflectance. Particularly, the method aims at finding the most Lambertian segment (sine-like) of an arrangement of pixel intensities, from which a recovered sine function can further help diminish the corrupted luminance response for each pixel. Our technique can be applied as a corrective pre-processing step in schemes working with photometric stereo or photometric sampling databases.

The article is organized as follows: in Section 1.1, we describe the previous work related to our approach; Section 2 introduces the decomposition of the luminance matrix and the proposed sine fitting technique to improve photometric consistency; Section 3 provides experimental evaluation, testing our method on both photometric sampling and photometric stereo databases; finally, conclusions and future work are outlined in Section 4.

\subsection{Related work}

The idea of fitting sine functions for shape recovery was originally proposed by Nayar et al. in [D], where they introduced the photometric sampling technique. Their method recovered shape by relating the sampling of a photometric function with surface orientation, reflectance and light sources. To this end, the authors proposed to measure (sample) the photometric response of a still object while a light source, placed on a turntable, moved around a single circular path.

Saito et al. [ㅁ] and more recently Liu et al. [四] borrowed the sine fitting idea of the photometric sampling to estimate the surface normals of a specular object. Assuming knowledge of the light source direction, the former described a least-squares fitting procedure applied on each RGB channel in order to avoid specular regions and calculate surface normals. A similar procedure using the Hough transform was proposed by the latter.

Miyasaki and Ikeuchi studied the effect of identifying shadows and specular reflections as outliers [ $\square]$. Aided by the Singular Value Decomposition (SVD) and the graph-cut algorithm, the method excluded outliers from the database before performing PSM, i.e., the pixels in the image that contained the outliers were removed to later apply SVD in a submatrix. A robust PCA approach for removing specularities and shadows in photometric stereo databases was also proposed in [ $[$ ].

Compared to previous work, our method considers the sampling of several circles around the object instead of using a single circular patch. For this reason, the construction of a luminance matrix is required. We propose to fit sine functions onto the singular vectors of this matrix rather than performing fitting onto the raw luminance data.

It is important to consider that our work seeks to provide a tool for diminishing photometric consistency departures in databases, so that other approaches aimed at surface shape recovery, light sources direction estimation, and specularity and shadow removal may benefit from a correcting database pre-processing step. 


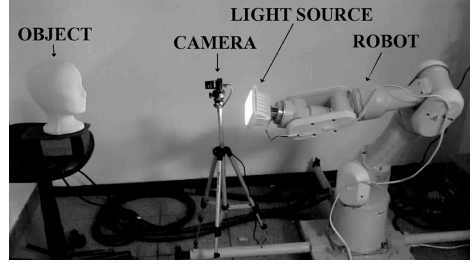

(a) Experimental setting

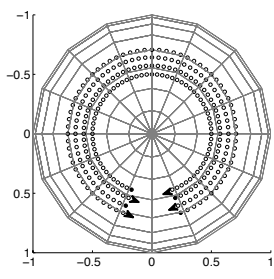

(b) Illumination patterns

Figure 1: Data acquisition process scheme for the photometric sampling database. In (a), the elements of the photometric sampling database acquisition scheme are shown, while (b) presents a projection of the light source positions, for four zenith and sixty-five azimuth angles. Separation between samples was $5^{\circ}$.

\section{Photometric consistency and correction}

This section explains the concept of photometric consistency and a sine fitting procedure introduced to improve the consistency of photometric databases. The main idea of our method is to analyze the luminance response at each pixel in order to locate a Lambertian-like behavior on the singular vectors of the luminance matrix. Once the most Lambertian patch is located along each singular vector, a sine function is fitted on the patch and the singular vectors replaced with the fitted functions. The procedure is described more in detail in the next pages.

\subsection{Photometric consistency}

In order to introduce the concept of photometric consistency let us start with Figure 1, where the data acquisition process for the photometric sampling database developed in our lab is depicted. The process was performed by a Mitsubishi PA-10 robotic arm to generate the illumination changes in accurate concentric patterns around the optical axis of the camera. A halogen lamp was attached to the end effector of the robot as shown in Fig. 1 (a). An example of the illumination patterns applied to the data acquisition process are plotted in Fig. 1 (b). For two different surfaces (a mannequin and a human face), a database of $k=k_{1} \times$ $k_{2}$ images of the observed object under $k$ different light source directions was constructed, with $k_{1}$ azimuth angles and $k_{2}$ zenith angles of the light source direction vector. Since the illumination changes in zenith away from the camera view usually provoke large shadowed regions, the zenith sampling was not extended beyond $30^{\circ}$. It is important to remark that, due to the concentric circle patterns, the set of sampled images should reflect the two main variations (i.e., changes related to azimuth and zenith) of the light source direction vector at each trajectory point.

We use the term photometric consistency to refer to the agreement of the captured images with the illumination changes imposed on the observed object. In other words, if the arrangement of a set of light sources is not arbitrary (supposes certain periodicity), the recorded image database should reflect the illumination patterns of such lighting arrangement. In Lambertian reflectance, circular lighting patterns lying on the surface of a sphere, and pointing towards the observed object, generate a perfect sinusoidal function (excluding shadowed regions) along each pixel value. Since most real surfaces do not respect Lambert's laws, the recorded luminance at each pixel will depart from the smoothness and shape of a sinusoidal signal. Consistency departures may be explained as phenomena inherent to the data 


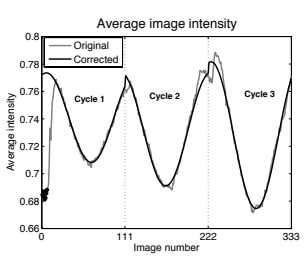

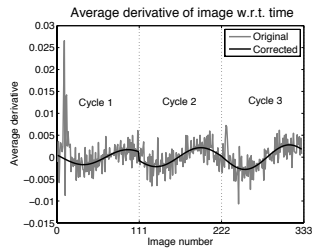

(a) Mannequin
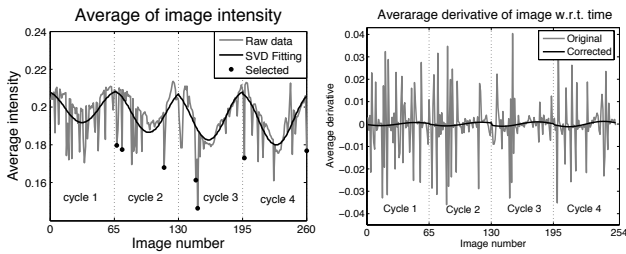

(b) Human face

Figure 2: Photometric consistency. The average image intensity and the average derivative of image with respect to time are shown as a measure of photometric consistency for the 333 and 260 images of each database respectively, before and after performing correction on the singular vectors of the luminance matrix. The average intensity plots include several dark circles, corresponding to those images whose departure from photometric consistency is the greatest.

acquisition conditions, i.e, noise, inter-reflections, high or low exposure, varying light source intensity, specular and shadow regions, and even slight movements of the object (in the case of a human subject). In our method, we aim to project such departures onto sine functions by a sine fitting strategy on the singular vectors of a luminance matrix, improving photometric consistency of the database and therefore its suitability for photometric analysis.

Figure 2 presents the average image intensity and the average derivative of image with respect to time as measures of consistency. Results after applying our method on a database of a styrofoam mannequin and a human face are presented in (a) and (b), respectively. The mannequin was matte painted in order to emulate Lambertian reflectance. The figure shows both consistency measures before (gray line) and after (dark line) applying photometric correction. In the figure, each cycle represents a change in zenith angle of the illumination pattern. Note how the human subject presents greater consistency departures than the matte mannequin, while our method achieves projection of the average image intensity to a sinelike function in both cases. Additionally, Figure 2 shows with dark circles those images (or light source directions) whose departure from photometric consistency was the greatest. For the case of the mannequin, the least consistent lighting arrangement corresponds to the starting points of the light source while the human subject reveals departures during all the acquisition process. More detailed explanation about this figure will be later provided using specific examples in the experimental section.

\subsection{Photometric correction}

We start this subsection by briefly describing the concept of photometric sampling, as it is important for understanding the photometric correction step. The principle of photometric sampling is based on the image irradiance equation for a Lambertian surface, that establishes the relationship of the surface normals $\mathbf{n}(u, v) \in \mathbb{R}^{3}$ and the light source direction $\mathbf{l}(u, v) \in \mathbb{R}^{3}$ to calculate the luminance for each pixel in the image: $i(u, v)=\langle\mathbf{n}, \mathbf{l}\rangle$.

In accordance with the gathered images during the data acquisition process, the luminances of a pixel will draw a sinusoidal function if the illumination variations imposed around the object are circular, i.e., the different light source direction vectors are circles lying on the surface of a virtual sphere. The sine curve can be decomposed in the three parameters: amplitude $(\mathbf{A})$, phase $(\mathbf{B})$ and shift $(\mathbf{C})$ as $I(\theta)=\mathbf{A} \sin (\theta+\mathbf{B})+\mathbf{C}$, where $I(\theta)$ is the pixel luminance at each $\theta$ variation in azimuth.

The photometric correction commences by generating, for each pixel, a matrix $\mathbf{M}_{k_{1} \times k_{2}}$ 

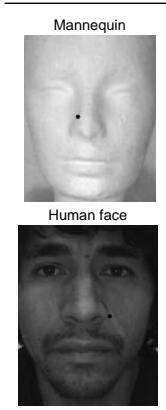
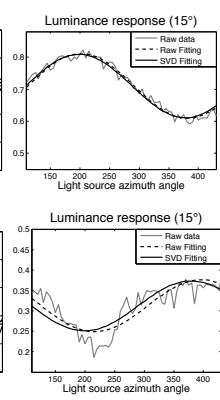
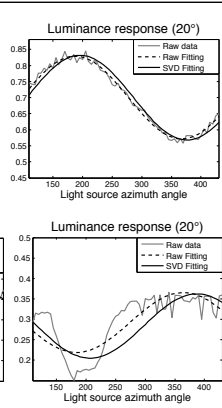
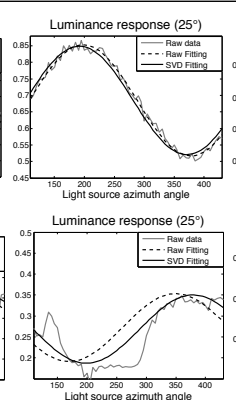
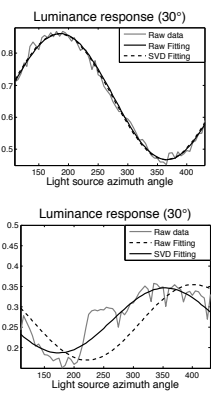

Figure 3: Photometric correction for two different reflectance examples. A mannequin and a human face are respectively shown in the top and bottom rows of the figure. For each case, the values in the luminance matrix for a single pixel (marked with a dark dot) are plotted in the second column. The rest of the columns depict sine fitting results on each vector of the luminance matrix.

storing the $k$ pixel intensity values recorded at each (azimuth, zenith) configuration pair. This matrix, which we refer to as the luminance matrix, contains the pixel reflectance history along the two main variations of the light source trajectory. For every pixel, the observed reflectance may be decomposed by the principal axis of the luminance matrix. The study of these axis allows identifying regions which best fit a sinusoidal behavior, i.e., close to a Lambertian behavior. The signal is finally corrected once the sine curve parameters have been calculated and the signal replaced with a sine function. We use SVD to decompose the luminance matrix,

$$
\mathbf{M}=\mathbf{U}_{k_{1} \times r} \Sigma_{r \times r} \mathbf{V}_{r \times k_{2}}^{T},
$$

where $r=\operatorname{rank}(\mathbf{M})$. The column and row spaces of $\mathbf{M}$ are decomposed into the orthogonal basis $\mathbf{U}$ (left singular vectors) and $\mathbf{V}$ (right singular vectors), respectively. The singular values, contained in the diagonal elements of $\Sigma$ explain the degree of retained variability in both right and left singular vectors. In our context $\mathbf{U}$ is related to the azimuth variations while $\mathbf{V}$ refers to variations in zenith.

Figure 3 shows a visual sketch of the light source trajectory variations over a surface normal of the mannequin and the human face. The location of the surface normal is indicated with a black dot in the first column of the figure. The second column of the figure shows a plot of the four columns (as four zenith angles were used) of the luminance matrix associated with the analyzed surface normal. Note how the reflectance of the mannequin at the selected pixel clearly exhibits a sinusoidal behavior compared to the human reflectance example.

The underlying idea of the image correction method proposed in this article is to find a suitable representation for maximizing photometric consistency in photometric databases. To this end, we studied the effect of performing sine fitting in the singular vectors of the luminance matrix of all image pixels along the database. The singular vectors of the luminance matrix are preferred against the raw column vectors of the matrix since artifacts or outliers are likely to be filtered out due to the weighting of singular values. As opposed to previous work $[\square, \square]$, where fitting sine functions on the raw data was used to determine surface shape assuming knowledge of the light source direction, our method aims to correct photometric consistency departures through fitting sine functions on the singular vectors of a luminance matrix.

Since real images may include noisy variations in reflectance (i.e., bottom row in Figure 3), using least squares for calculating the three sine parameters (amplitude, phase and 

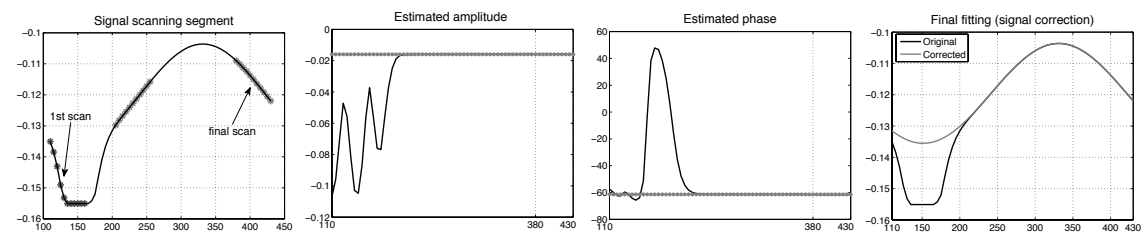

Figure 4: The sinusoidal fitting procedure. The figure illustrates the proposed sine fitting procedure, based on scanning sine segments through the singular vectors of the luminance matrix.

shift) may lead to poor estimations in the sought parameters, as behaviors departing from Lambert's law may occupy a large region of the singular vector. To overcome this problem, we performed fitting using smaller fixed-sized signal periods starting from each point along the singular vector, then chose the phase, amplitude and shift parameters appearing in the majority of the cases. The fitting procedure is roughly illustrated by Figure 4. In the figure, the leftmost diagram exemplifies the scanning path for a segment of 50 degrees length (ten separations of five degrees each) of an arbitrary pixel for a synthetic sphere. The second and third diagrams respectively show the estimated amplitude and phase after fitting a sinusoidal signal onto each segment. Note how, for each segment scan, the obtained amplitude, phase and shift are recorded separately. Once all the parameters have been gathered for each scan evaluation, a single value for each parameter is decided (using the mode interval) and the complete signal fitting finally performed. This procedure applies to all left and right singular vectors whose singular value is greater than zero. Finally, the rightmost diagram of Figure 4 shows the fitting result after calculation of the three optimal coefficients.

Once the sine parameters are estimated over each of the singular vectors, a new luminance matrix $\mathbf{M}^{\prime}=\mathbf{U}^{\prime} \Sigma \mathbf{V}^{\prime T}$ is generated to improve photometric consistency on the database. The new fitted columns of $\mathbf{U}^{\prime}$ and $\mathbf{V}^{\prime}$ contain the sine-fitted singular vectors from the original matrices $\mathbf{U}$ and $\mathbf{V}$ in Eq. 1 .

Let us turn our attention back to Figure 3, where the last four columns show the sine fitting results on the singular vectors of the luminance matrix (solid lines) and the sine fitting results on the raw data of the luminance matrix (dotted lines). This comparison is provided in order to motivate the singular vectors as an appropriate representation for image correction. The raw data of the luminance matrix is shown in gray for the four cases. The same fitting strategy shown in Figure 4 was applied to the raw data. Each luminance response is presented separately so as to isolate the variations in zenith angle, which varies from $15^{\circ}$ to $30^{\circ}$. Note how, for the mannequin responses, there seem to be no major difference in fitting the sine functions to the singular vectors. This may be due to the matte reflectance of the surface. Nonetheless, the reflectance responses of the human face indicate a more accurate fitting for the singular vectors (solid line), specially when the zenith angle departs from the camera direction (right-most columns of Figure 3).

In order to motivate the singular vectors of the luminance matrix as an appropriate representation for photometric consistency, we additionally performed the following tests. In a first test, 200 subsets were randomly selected from the mannequin database, before and after correction on both the singular vectors and the raw columns of the luminance matrix. All the subsets contained seven images from the $65 \times 4$ images in the database. Each image in the subset was transformed into a long column vector to form a long matrix of seven columns and SVD was applied to these matrices. Similar to [0], the first three left singular vectors of this matrix were used to build a surface normal map, later integrated to obtain a corre- 


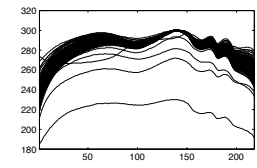

(a) Non-corrected.

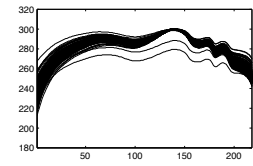

(b) Raw data fitted.

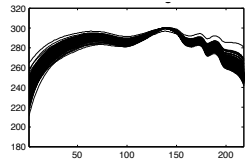

(c) Sing. vector fitted.

Figure 5: Recovered profiles for the mannequin. The figure reveals an improved consistency in shape recovery after fitting the sinusoidal function on the singular vectors of the luminance matrix.

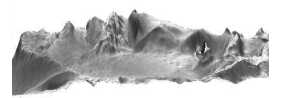

(a) Non-corrected.

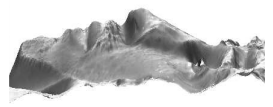

(b) Raw data fitted.

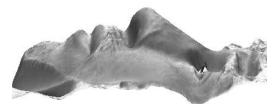

(c) Sing. vector fitted.

Figure 6: Surface recovery for a human face. Results after performing correction on the singular vectors of the luminance matrix provide stability and smoothness to the recovered surface.

sponding surface. The Frankot and Chellappa integration method [ [] with Discrete Cosine Transform (DCT) basis functions was used for the integration step. The results of this experiment are shown in Figure 5, where the profiles of the recovered surfaces are shown for the 200 subsets. From the figure, the shape variation around the profiles reveals difference in consistency among the three databases. Although the surface is nearly Lambertian, the original data profiles in (a) reveal a major disagreement in surface estimations. The raw data fitted profiles in (b) present some surfaces out of the regular estimations, while in (c) the consistency of the database suggests an improvement along the 200 integrated surfaces.

A second test presents shape recovery results on the human face database. We selected the seven images with the biggest departure from the raw and corrected average image intensity, which are marked with a dark spot in Figure 2(b). For each subset, surface shape recovery was achieved as formerly explained in the previous test. Figure 6 presents the surface reconstruction results from non corrected data (a), from sine fitting onto the raw data (b) and from sine fitting onto the singular vectors of the luminance matrix (c). The figure reveals that the image correction clearly benefits the photometric properties of the database, more particularly, performing sine fitting to the singular vectors of the luminance matrix provides more symmetrical and smooth results than only fitting sines directly to the luminance matrix.

From figures 5 and 6 we can observe that sine fitting on the singular vectors of the luminance matrix provides an advantage over sine fitting on the raw luminance data, particularly for non-Lambertian reflectance. This fact supports the underlying idea of our method, i.e., to remove photometric consistency departures in photometric databases by using an appropriate decomposition of the surface normal reflectance.

\section{Experimental results}

We start this section describing results from the photometric sampling database. Figure 7 depicts, with two main panels, for the mannequin and human face cases, the subset of original and corrected images used for surface shape recovery followed by surface normal integration. The lighting configuration, shown at the left side of the panels, corresponds to those images with the greatest consistency departure in the database, i.e., the dark circles shown in Figure 2. The image difference is also provided in both panels. Note how, for the mannequin example, the proximity of the light source directions makes it practically impossible to notice the 

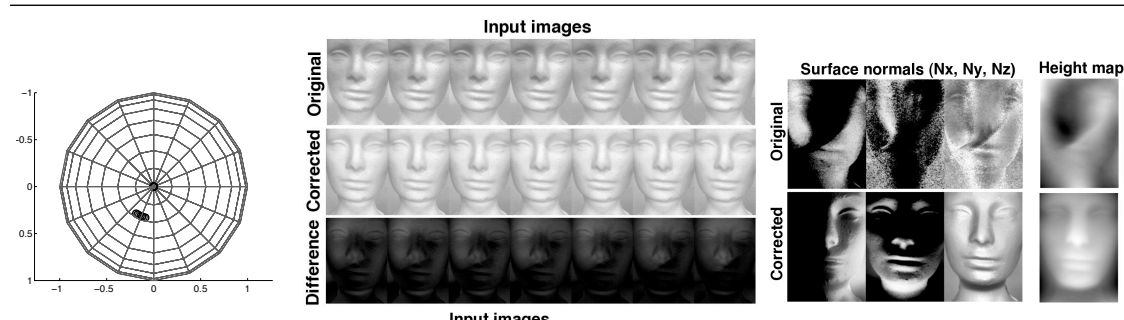

Surface view

Input images
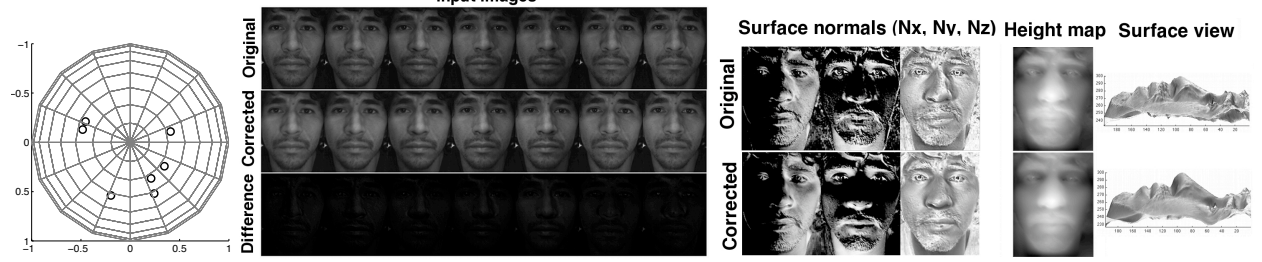

Figure 7: Experimental results on the photometric sampling database. The figure presents original and corrected results for the selected image subsets shown as dark circles in Figure 2.

illumination variation from the visual inspection of the image subsets, while the difference between original and corrected images reveals the remotion of shadowed areas provoked by occluded areas between the light with the camera tripod. The benefit from photometric correction is noticeable by visually comparing the shape recovery results. Particularly, the remotion of shaded areas allows statistical PSM to determine the contribution of each pixel in the orthogonal image decomposition, while the smoothness of the results suggest numerical consistency of the SVD. This result is opposed to the spurious noise specially present in the background of the surface normals obtained from the non-corrected images. As far as the human face example is concerned, the perceptual difference between the original and corrected images is corroborated by its image difference. Note how the original images appear darker than their corrected counterparts, which suggests that inter-reflections are diminished by homogenizing the overall brightness of the dataset. Again, the image differences suggest that main modifications after correction are related to imposing consistency for illumination changes. This fact is supported by the surface shape recovery results of the figure. In this case, the $y$-axis of the surface normals seem to get the biggest benefit from our method.

We also tested our method using a photometric stereo database. The extended Yale B database of faces [ $[\mathrm{Q}]$ has been acquired using a spherical structure with 64 illumination variations for images of human faces. We have chosen this database because its spherical setting is similar to the one utilized in the photometric sampling experiments. For all the cases, we used the two first zenith angles (concentric circles) of the database, i.e., the luminance matrix size was $6 \times 2$, considerably smaller than the previous examples on a photometric sampling database. Once the sine fitting procedure depicted in the previous section was applied on the database, we picked the innermost six image subset in order to recover shape. Following the theory of [ $[$ ] , the arrangement of the light sources in this subset is adequate since it varies uniformly around the azimuth angle and keep its zenith angle fixed. Shape recovery results are shown before and after (singular vector) correction in Figure 8. From top to bottom, the figure presents results organized in three panels, for subjects 11,13 and 16 of the database. Each panel depicts the subset of original and corrected images, its image difference, estimated surface normals and estimated shape from the subset.

For subject 11 (top panel), a visual comparison of the original and corrected images 

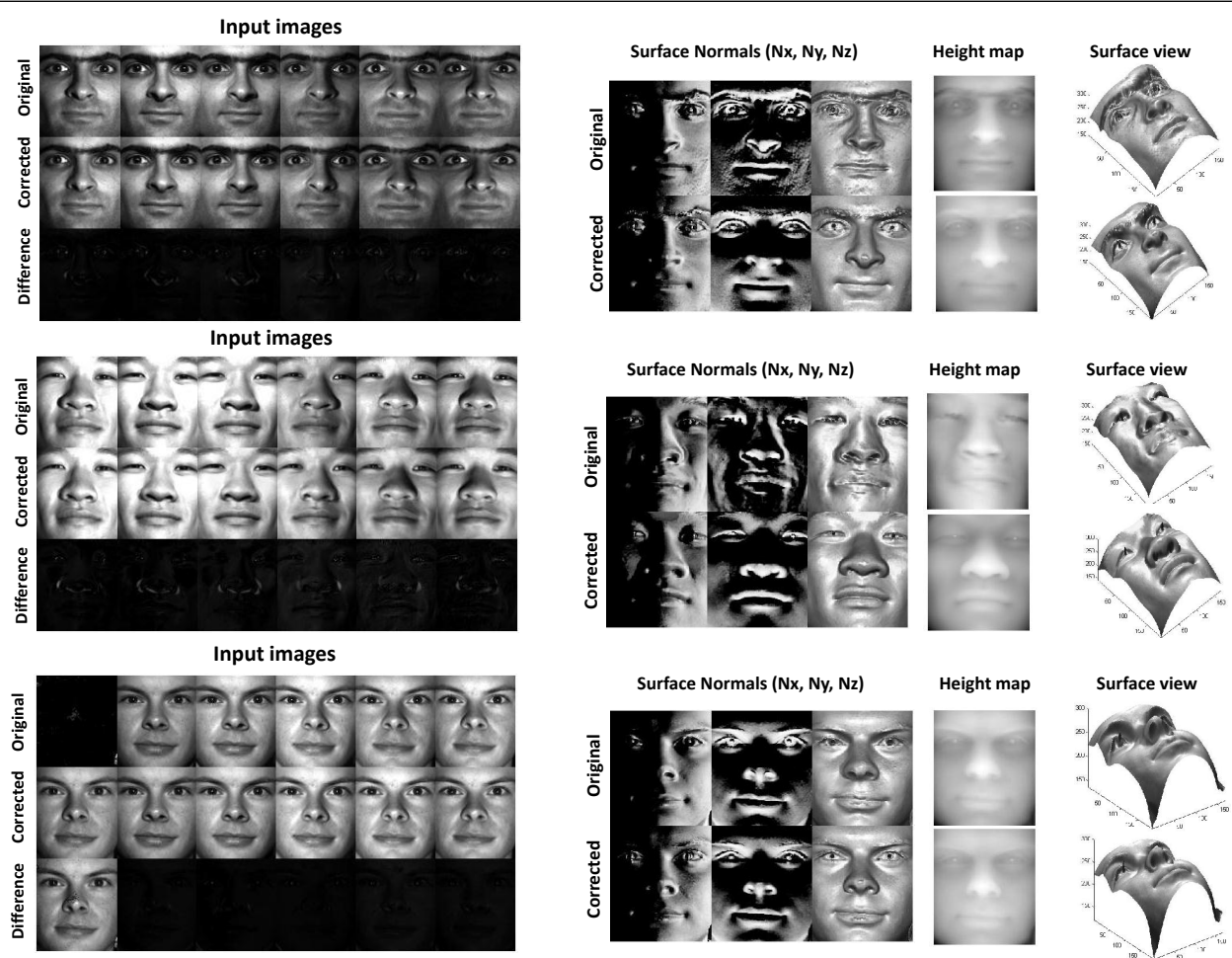

Figure 8: Experimental results on a photometric stereo database. Original and corrected imagery and their corresponding surface shape estimation results are presented for three subjects of the extended Yale B database.

reveals imperceptible differences, located mainly around the nose and eyes of the subject. Despite this negligible difference, the surface recovery is greatly improved for the corrected data. This can be observed in the overall shape of the surface, which improves smoothness and stability of fine details around the nose and the eyes. Moreover, the eyebrow area, which appears concave in the estimated surface from the non-corrected data, becomes convex for for the corrected case.

The visual inspection of the middle panel (subject 13) reveals a more noticeable difference between original and corrected images. This difference, majorly located around the nose area might suggest a slight movement of the subject during the data acquisition process. Note also how the third, fourth and fifth image of the subset present a difference in exposure and, in general, the corrected images appear more uniformly illuminated than the original subset. These observations are corroborated through the estimated surface normals and shape recovery for both original and corrected data, where the latter delivers greatly improved results, i.e., the instabilities of the surface, possibly caused by the movement of the subject, are considerably removed.

Finally, the bottom panel of Figure 8 (subject 16) presents an alternative application of our method, since one of the images of the subset is severely corrupted, almost missing, due to problems in the acquisition process. Note how the proposed photometric correction is capable of recovering the corrupted image. Other improvements can be noticed in the 
surface recovery, around the eyebrow and nose areas.

\section{Conclusions}

We have introduced an approach for image correction in photometric databases, based on a sine signal fitting on the singular vectors of a luminance matrix. Experiments demonstrate that the proposed correction is useful to improve image quality and to provide consistency on the lighting variation along the database. Future work will address the implementation of alternative sine fitting methods (RANSAC, Hough transform) to compare efficiency and performance, as well as the suitability of the method to be incorporated into particular photometric shape recovery frameworks.

\section{References}

[1] V. Argyriou and M. Petrou. Recursive photometric stereo when multiple shadows and highlights are present. In Proc. CVPR, pages 1-6, 2008.

[2] S. Barsky and M. Petrou. The 4-source photometric stereo technique for three-dimensional surfaces in the presence of highlights and shadows. IEEE Trans. Pattern Anal. Mach. Intell., 25(10): 1239-1252, 2003.

[3] E. J. Candès, X. Li, Y. Ma, and J. Wright. Robust Principal Component Analysis? ArXiv e-prints, December 2009.

[4] O. Drbohlav and M. Chantler. On optimal light configurations in photometric stereo. In ICCV'05, pages 1707-1712, 2005.

[5] R. Frankot and R. Chellappa. A method for enforcing integrability in shape from shading algorithms. IEEE Trans. Pattern Anal. Mach. Intell., 10:438-451, 1988.

[6] A. Georghiades, D. Belhumeur, and D. Kriegman. From few to many: Illumination cone models for face recognition under variable lighting and pose. IEEE Trans. Pattern Anal. Mach. Intell., 23(6):634-660, 2001.

[7] C. Hernández, G. Vogiatzis, and R. Cipolla. Shadows in three-source photometric stereo. In Proc. ECCV, 2008.

[8] J. Ho, M. Yang, J. Lim, K. Lee, and D. Kriegman. Clustering appearances of objects under varying illumination conditions. In Proc. IEEE Conf. on Computer Vision and Pattern Recognition, pages 11-18, 2003.

[9] K.C. Lee, J. Ho, and D. Kriegman. Acquiring linear subspaces for face recognition under variable lighting. IEEE Trans. Pattern Anal. Mach. Intelligence, 27(5):684-698, 2005.

[10] R. Liu and J. Han. Recovering surface normal of specular object by hough transform method. Computer Vision, IET, 2010.

[11] D. Miyasaki and K. Ikeuchi. Photometric stereo under unknown light sources using robust svd with missing data. In Proc. ICIP, 2010.

[12] S.K. Nayar, K. Ikeuchi, and T. Kanade. Shape and reflectance from an image sequence generated using extended source. Proceedings of IEEE ICRA, 1:28-35, 1989.

[13] H. Saito, K. Omata, and S. Ozawa. Recovery of shape and surface reflectance of specular object from relative rotation of light source. Image and Vision Computing, 21:777-787, 2003. 